\author{
Mediterranean BioMedical Journals \\ Integrative Journal of Medical Sciences \\ 2020, Volume 7, ID 153 \\ DOI: $10.15342 / \mathrm{ijms.7.153}$
}

LETTER TO THE EDITOR

\title{
Will COVID-19 Drive Innovation within Medical Education and Residency Training?
}

\author{
Youssef Kharbach (D); Abdelhak Khallouk
}

Urology department, Tangier University Hospital

Faculty of Medicine and Pharmacy, Abdelmalek Essaâdi University

9000 Tangier, Morocco

Correspondence : Dr Youssef Kharbach, Urology department, Tangier University Hospital. Faculty of Medicine and Pharmacy, Abdelmalek Essaâdi University.9000 Tangier, Morocco. Email : ykharbach@uae.ac.ma

Copyright (C) 2020 Youssef Kharbach and Abdelhak Khallouk

This is an open access article distributed under the Creative Commons Attribution 4.0 International, which permits unrestricted use, distribution, and reproduction in any medium, provided the original work is properly cited.

Dear Editor,

Coronavirus disease 2019 (COVID-19) is now declared as a Public Health Emergency of International Concern by the The World Health Organization (WHO)[1]. Thus, on March 22 the Moroccan government adopted the texts relating to the implementation of the state of health emergency including quarantine and physical distancing [2]. All this disrupted routines in hospitals and medical schools. Subsequently, students and faculty should adapt to this unpredictable situation.

Medical education is specific with both clinical clerkships in the mornings and lectures at the faculty in the afternoons. During the COVID-19 pandemic, medical students were facing with a serious dilemma: on the one hand, their learning is not yet complete and should keep clinical clerkships to maintain learning, on the other hand, their lack of knowledge make them potential vectors for COVID-19. Meanwhile, several teaching hospitals all over the world have reported cases of COVID-19 in medical students [3].

Thus, the Moroccan government decided to suspend clinical clerkships. This decision seems to be wise as for example, many medical schools around the world cancelled formal teaching on wards [3,5]. However, many of our students were frustrated by this decision because they were willing to assist in the care of patients with COVID-19 in the hospital.
By the application of physical distancing during the pandemic, including Technology Enhanced Learning (TEL) in medical education became mandatory. Our medicine faculty had quickly transitioned the entire medical education program to online formats to prevent delays in educational progress. We offered to our students recorded lectures and pdf documents. And currently, we are developing live-streams. This situation will be a great opportunity for our students to adapt with new technologies and prepare the implementation of telemedicine, which is a necessity in our country where medical need is still not totally satisfied.

Our students responded well to online learning as most of them belong to the Millennial generation and are adept in the use of technology-enhanced learning resources. Similar conclusions are found in the literature [5,6]. Also, it is known that the lack of interactivity could be a real barrier to recognize students' real needs. So, we created an online forum to have students' feedback and answer their questions to make lectures as interactive as possible. Actually, there are many technologies that could be used in medical education. Implementation of technologies and online problem-based learning techniques is crucial to help students to develop necessary medical skills in a new manner [4].

Massive open online courses (MOOCs) are of great interest in this situation. Swennerton et al. [7] showed that three-week anatomy MOOC was well received by 
medical students with high level of usage. The course was used to support the students' consolidation and revision. Even if the students appreciated the MOOC content, a significant majority of them did not want the MOOC to replace the existing teaching they receive [7], suggesting that students still highly value the time spent with the teaching staff.

However, the online learning creates a loss of collaborative experiences that could disturb medical education [4]. Also, interrupting clinical clerkships makes our students lose opportunities for personal development which have the potential to be a serious detriment to medical students' career trajectory [4].

Residents' training is also affected. All hospital activities were drastically reduced and limited to emergencies and non-deferrable procedures. The procedures affected by these limitations are those where the residents are mostly involved [8]. Moreover, residents were deployed as primary care doctors in emergency services or in COVID-19 screening units because of the increasing need to health workers.

As urology professors, we have the mission of maintaining high-quality resident education. We should innovate in our teaching. Also, our ability to provide mentorship to our residents conducted us to prepare them to practice medicine on the frontlines of the crisis. In order to limit the impact of the pandemic on residents' learning, Porpiglia et al. [8] suggest different types of smart-learning technologies such as : pre-recorded videos, wibinars, podcasts and « virtual » clinical staff

\section{ABBREVIATIONS}

COVID-19: Coronavirus disease 2019

WHO: The World Health Organization

MOOC: Massive open online courses

TEL: Technology Enhanced Learning

\section{COMPETING INTERESTS}

The authors declare that there is no conflict of interest.

\section{FUNDING}

This research received no specific grant from any funding agency in the public, commercial, or not-for-profit sectors.

\section{REFERENCES}

[1] WHO. Coronavirus disease (COVID-19) Pandemic. https://www.who.int/emergencies/diseases/novelcoronavirus-2019. Accessed May 7, 2020.

[2] Moroccan government. https://www.cg.gov.ma/fr/conseilsde-gouvernement/le-gouvernement-adopte-les-textes-relatifsa-la-mise-en-oeuvre-de-letat-d. Accessed May 7, 2020.

[3] Ahmed H, Allaf M, Elghazaly H. COVID-19 and medical education. Lancet Infect Dis 2020;S1473-3099(20)30226-7.

[4] Ferrel MN, Ryan JJ. The Impact of COVID-19 on Medical Education. Cureus 2020;12:e7492.

[5] Pickering JD, Swinnerton BJ. Exploring the Dimensions of Medical Student Engagement with Technology-Enhanced Learning Resources and Assessing the Impact on Assessment Outcomes. Anat Sci Educ 2019;12:117-28. and rounds. This could ensure continuity in residents' learning especially since the duration of the pandemic is unpredictable.

Also, conferences, seminars and congresses meetings attendance are also essential components of residency training. But, physical distancing measurements and the increased spread of COVID-19 had led to the cancellation of numerous such scientific events.

This situation presents an adaptability challenge for our students and residents. Their mental health is significantly affected making psychological assistance of great importance. The panic is palpable; they are worried about learning quality and exams whose modalities are not yet clear. A recent study Cao el al. [9] showed that $24.9 \%$ of college students have experienced anxiety because of the COVID-19 pandemic. They suggest that the students' mental health should be monitored. Also, they recommend crisis-oriented psychological services to students to reduce the psychological pressure [9].

In conclusion, the real impact of the pandemic on students and residents learning is still unknown. However, technology gave us the possibility to ensure correct medical education. It required lots of hard work. Pandemic had improved the adaptability of medical students and residents but also led professors to be innovative in teaching. Professors of our faculty are sharing their practices with their peers to satisfy graduation requirements on-time and achieve quality online learning.

\section{ACKNOWLEDGMENTS \\ None}

\section{AUTHORS' CONTRIBUTIONS}

The participation of both authors corresponds to the criteria of authorship and contributorship emphasized in the Recommendations for the Conduct, Reporting, Editing, and Publication of Scholarly work in Medical Journals of the International Committee of Medical Journal Editors. Indeed, both authors have actively participated in the redaction, the revision of the manuscript and provided approval for this final revised version

[6] Evans DJR, Bay BH, Wilson TD et al. Going Virtual to Support Anatomy Education: A STOP GAP in the Midst of the Covid-19 Pandemic. Anat Sci Educ 2020;10.1002/ase.1963.

[7] Swinnerton BJ, Morris NP, Hotchkiss S et al. The integration of an anatomy massive open online course (MOOC) into a medical anatomy curriculum. Anat Sci Educ 2017;10:53-67.

[8] Porpiglia F, Checcucci E, Amparore D, et al. Slowdown of urology residents' learning curve during COVID-19 emergency. BJU Int 2020;10.1111/bju.15076.

[9] Cao W, Fang Z, Hou G, et al. The psychological impact of the COVID-19 epidemic on college students in China. Psychiatry Res 2020;287:112934. 